\title{
Age-Related Changes of Calbindin D-28k-Immunoreactive Neurons in the Myenteric Plexus of Gerbil Duodenum
}

\author{
Jung Hoon $\mathrm{CHOI}^{1)}$, Choong Hyun $\mathrm{LEE}^{1)}$, Dae Won $\mathrm{CHUNG}^{1)}$, In Koo HWANG ${ }^{1)}$, Moo Ho WON ${ }^{2)}$, Je Kyung SEONG ${ }^{1)}$, \\ Yeo Sung $\mathrm{YOON}^{1)}$ and In Se $\mathrm{LEE}^{1) *}$ \\ ${ }^{1)}$ Department of Anatomy and Cell Biology, College of Veterinary Medicine and BK21 Program for Veterinary Science, Seoul National \\ University, Seoul 151-742 and ${ }^{2}$ Department of Anatomy and Neurobiology, College of Medicine, Hallym University, Chuncheon 200 \\ 702, South Korea
}

(Received 24 July 2007/Accepted 7 December 2007)

ABSTRACT. We examined the age-related changes of calbindin D-28k (CB)-immunoreactive neurons and overall populations of neurons in the myenteric plexus of gerbil duodenum using whole mount preparations and immunohistochemistry. The circumference of duodenum increased age-dependently. CB-immunoreactive neurons were observed in all groups, and most of them had the Dogiel type II morphology. The fully developed cobweb-like structures were observed in the myenteric plexus of duodenum at postnatal month (PM) 3 to 24. Although the highest numbers of CB-immunoreactive neurons and overall population were observed in PM 1.5, it is related with significant increase of the size of circumference between PM 1.5 to PM 3. CB-immunoreactive neurons were slightly decreased with age between PM 3 to PM 24. We have also found that whole numbers of myenteric neurons were also significantly decreased in PM 24 group. These results suggest that loss of overall numbers of myenteric neurons and CB-immunoreactive neurons may be related with age-related neurodegeneration and functional loss of duodenum in the gerbil.

KEY WORDS: aging, calbindin, duodenum, gerbil, myenteric plexus.

The autonomic nervous system is divided into two divisions, e.g. parasympathetic nervous system and sympathetic nervous system. In recent decade, the enteric nervous system (ENS) is the largest division of the autonomic nervous system, because it can perform its function independently from the central nervous system [7, 20, 44]. It consists of local nerve networks embedded in the gut wall and is subdivided into two ganglionated plexuses, i.e., the submucosal and myenteric plexuses $[40,44]$. The ENS mediates complex reflex activities involving intestinal motility, mucosal transportation and blood flow [7]. In electrophysiological studies, the majority of the enteric neurons could be placed in one of two classes: synaptic neurons or after-hyperpolarizing $(\mathrm{AH})$ neurons [21, 22, 26, 29, 34].

The myenteric plexus lies between the longitudinal and circular muscular layers and extends entire length of the gut. It has been known that the myenteric plexus primarily provides motor innervation to the two muscular layers and secremotor innervation to the mucosa $[15,44]$.

Calbindin D-28k (CB), one of the calcium binding proteins, plays an important role as an intracellular facilitator of calcium diffusion or as a modulator of neurotransmission [3, $4,16,17]$. In neurons, action potentials are also carried in part by inward calcium current [22, 26, 34]. It has been reported that $\mathrm{AH}$ neurons were sensory neurons because these neurons lacked fast excitatory synaptic inputs $[21,22$, $26,29,34]$. More recently, with the help of the combination of the electrophysiological and immunohistochemical meth-

\footnotetext{
* Correspondence to: Prof. Lee, I. S., Department of Anatomy and Cell Biology, College of Veterinary Medicine, Seoul National University, Seoul 151-742, South Korea.

e-mail: inselee@snu.ac.kr
}

ods, it has been identified that the AH neurons had Dogiel type II morphology [14, 18, 26].

Although the distribution of CB-containing neuronal system has been reported upon the forebrain [11, 23, 24], comprehensive knowledge about the maturation of $\mathrm{CB}$ neurons in myenteric plexus is limited. The development and aging of CB-immunoreactive neurons in myenteric plexus are critical for proper intestinal functions through the whole life of human and animals $[4,9,16,26,32]$. Therefore, in the present study, we examined the age-related changes of CBimmunoreactive neurons, their processes and whole populations of myenteric neurons in the gerbil myenteric plexus to establish aging patterns of neurochemically identified neurons in the duodenum.

\section{MATERIALS AND METHODS}

Experimental animals: Male Mongolian gerbils (Meriones unguiculatus) were obtained from the Experimental Animal Center, Hallym University, Chuncheon, South Korea. The animals were kindly supplied by Dr. Oh, a chief in the Experimental Animal Center at Hallym University. Postnatal month (PM) $1.5(n=10)$, PM $3(n=10)$, PM 6 $(n=10)$, PM $12(n=10)$ and PM $24(n=10)$ gerbils were housed in a conventional state under adequate temperature $\left(23^{\circ} \mathrm{C}\right)$ and humidity $(60 \%)$ control with a 12-hr light/12-hr dark cycle, and free access to food and water. The procedures for handling and caring for the animals adhered to the guidelines that are in compliance with the current international laws and policies (NIH Guide for the Care and Use of Laboratory Animals, NIH Publication No. 85-23, 1985, revised 1996). All experiments were conducted to minimize 
the number of animals used and the suffering caused by the procedures used in the present study.

Tissue processing: For the histological analysis, ten animals per each group were anesthetized with sodium pentobarbital and were perfused transcardially with $0.1 \mathrm{M}$ phosphate-buffered saline (PBS, $\mathrm{pH} 7.4$ ), followed by $4 \%$ paraformaldehyde in $0.1 \mathrm{M}$ phosphate-buffer (PB, $\mathrm{pH} 7.4)$. For morphometric analysis, the duodenum was opened along the mesentery and gently placed on saline-moistened filter paper; the circumference was measured with a ruler at various aged animals. Thereafter, the tissues were stretched and pinned flatly on pieces of balsa wood. The tissues were then postfixed with the same fixative with mucosal surface upward for $6 \mathrm{hr}$. Circumference of the duodenum was measured at least 5 points along the entire length. The preparations were prepared by dissecting apart the layers of duodenum with watchmaker forceps.

Cresyl violet staining for myenteric neuron: Cresyl violet staining was performed with the preparations to determine the density of ganglia and the number of neurons in the myenteric plexus of gerbil duodenum.

Immunohistochemistry for $C B$ : Immunohistochemistry was performed under the same conditions in gerbils of different ages in order to examine whether the degree of immunohistochemical staining was accurate [25]. The whole mount preparations were sequentially treated with $0.3 \%$ hydrogen peroxide $\left(\mathrm{H}_{2} \mathrm{O}_{2}\right)$ in PBS for 30 min and $10 \%$ normal goat serum in $0.05 \mathrm{M}$ PBS for $30 \mathrm{~min}$. They were then incubated with diluted mouse anti-CB antibody (diluted to 1:1,000; Code no: 300, Swant, Switzerland) overnight at room temperature and subsequently exposed to biotinylated goat anti-mouse $\operatorname{IgG}$ and streptavidin peroxidase complex (diluted to $1: 200$, Vector, U.S.A.). They were then visualized by staining with 3,3'-diaminobenzidine (Sigma, St. Louis, MO, U.S.A.) in 0.1 M Tris-HCl buffer ( $\mathrm{pH}$ 7.2) and mounted on gelatin-coated slides. They were mounted in Canada Balsam (Kanto, Japan) following dehydration. A negative control test was carried out using non-immune serum instead of primary antibody in order to establish the specificity of the immunostaining. The negative control resulted in the absence of immunoreactivity in any structures.

Quantification of data and statistical analysis: For quantitative analysis of CB-positive cells and myenteric neurons in the duodenum, at least 5 areas $\left(0.5 \mathrm{~mm}^{2}\right)$ per each animal were randomly selected. Count of the number of neuron was performed using an image analyzing system equipped with a computer-based CCD camera (software: Optimas 6.5 , CyberMetrics, U.S.A.) with $\times 20$ and $\times 40$ objectives.

Differences among means were statistically analyzed by one-way analysis of variance followed by the Duncan's new multiple range method or the Newman-Keuls test to elucidate the age-related changes of CB-immunoreactive neurons or to determine the age-related changes of circumference. $P<0.05$ was considered significant.

\section{RESULTS}

Age-related changes of circumference in duodenum: The circumference of duodenum was gradually increased in agedependent manner, and the significant change of their size was observed between PM 1.5 and 3 groups, thereafter slightly increased with age (Fig. 1).

Age-related changes of neuronal populations in duodenum: The highest density of myenteric neurons was detected in PM 1.5, and average numbers was $47.7 \pm 1.6$ per $0.5 \mathrm{~mm}^{2}$ area. There are no significant changes of neuronal populations between PM 3 to 12 groups. The neurons were counted with averages of 38.6 \pm 1.7 (PM 3), $37.4 \pm 0.7$ (PM 6 ), $37 \pm 0.9$ (PM 12) cells per unit area from 7 preparations in this group (Fig. 2). However, significant neuronal loss was found in PM 24 group compared with that of PM 3 to 12 groups. An average of the myenteric neurons in PM 24 was $32 \pm 0.8$ cells per unit area (Fig. 2).

Age-related changes of $C B$-immunoreactive neurons in duodenum: CB-immunoreactive nerve cell bodies and fibers were found in the myenteric plexuses of all age groups. In the whole mount preparations, $\mathrm{CB}$-immunoreactive neurons and their fibers were formed into cobweb-like structures in all age groups, however, the cell size of cobweb-like structure was increased age-dependently until PM 6 group (Fig. 3). The CB-immunoreactive cells displayed the Dogiel type II morphology, i.e., the cells were oval with generally smooth surfaces and gave rise to a number of long, gradually tapering processes, which ran predominantly in the circumferential direction. The varicosities of CBimmunoreactive nerve fibers were well-developed in the ganglia (Fig. 3F, G). There were no morphological differences of $\mathrm{CB}$-immunoreactive neurons with age.

In PM 1.5, we observed cobweb-like structures of the myenteric plexus in whole mount preparations, however, they seemed not to form into fully developed structures (Fig.

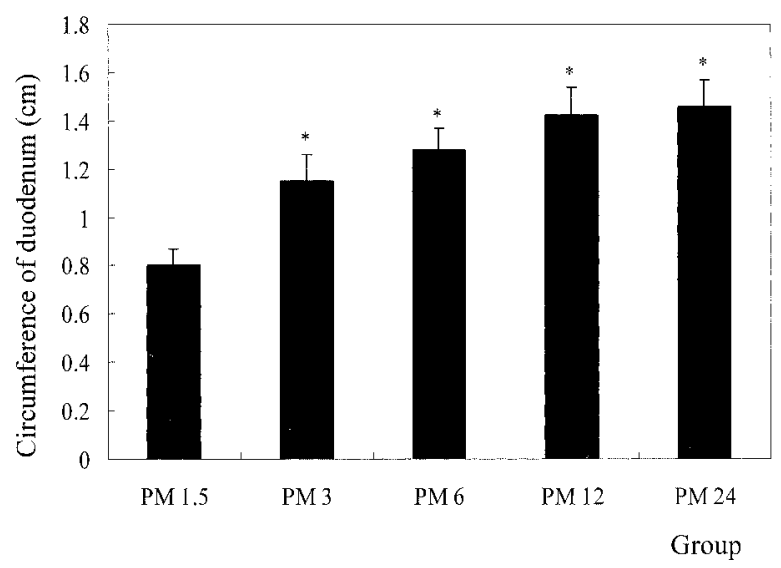

Fig. 1. Age-related changes of circumference in the gerbil duodenum. Differences among the means are statistically analyzed ( $* P<0.05$, significantly different from PM 1.5 group). The bar indicates the means \pm S.E.M. 


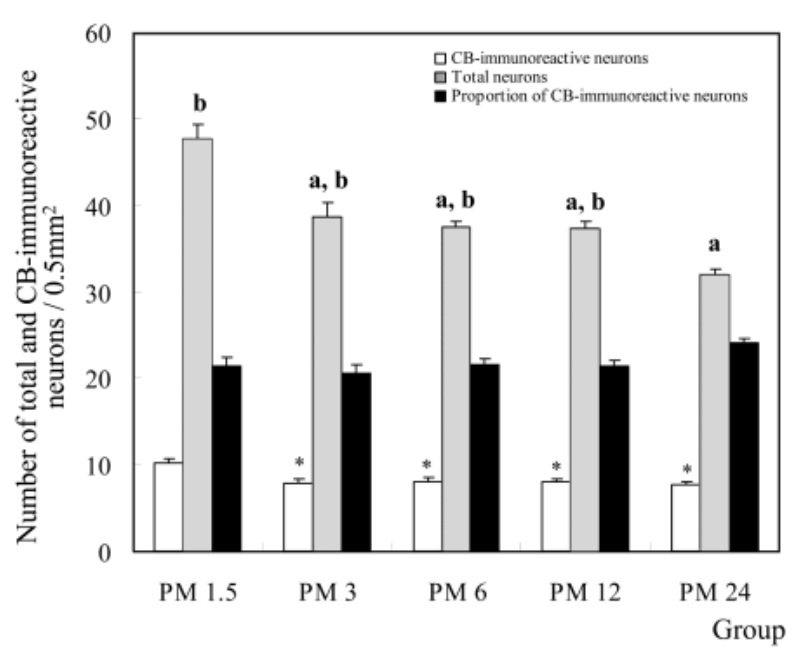

Fig. 2. Age-related changes of whole populations, calbindin D$28 \mathrm{k}(\mathrm{CB})$-immunoreactive neurons and proportions (\%) of CBimmuoreactive neurons of whole populations in the myenteric plexus of the gerbil duodenum. The data show the total number of CB-immunoreactive neurons per $0.5 \mathrm{~mm}^{2}$ area. Differences among the means are statistically analyzed $\left({ }^{\mathrm{a}} P<0.05\right.$, $* P<0.05$, significantly different from PM 1.5 group, ${ }^{\mathrm{b}} P<0.05$, significantly different from PM 24 group). The bar indicates the means \pm S.E.M.

3A). CB-immunoreactive neurons were counted with an average of $10.2 \pm 0.5$ cells per unit area $\left(0.5 \mathrm{~mm}^{2}\right)$ from 7 preparations in this group (Fig. 2).

In PM 3 group, the internodal strands were arranged and more developed. CB-immunoreactive varicosities were also detected in the myenteric plexus, however, their intensity was decreased (Fig. 3B). The number of CB-immunoreactive neurons significantly decreased more than that in PM 1.5 group (Fig. 2). The CB-immunoreactive neurons were counted with an average of $7.9 \pm 0.4$ cells per unit area from 7 preparations in this group (Fig. 2).

CB-immunoreactive internodal strands in PM 6 showed fully developed morphology, displaying more mature feature than that in previous stages (Fig. 3C). However, CB immunoreactivity was weaker than that of PM 3 group. In this group, the number of $\mathrm{CB}$-immunoreactive neurons was $8.1 \pm 0.5$ cells per unit area from 7 preparations (Fig. 2).

In PM 12 group, secondary component in internodal strands was detected (Fig. 3D). However, those CB-immunoreactive secondary internodal strands were thin and weak stained. In this group, the number of CB-immunoreactive neurons counted into $8.0 \pm 0.4$ cells per unit area from 7 preparations was similar that of previous stage (Fig. 2).

In PM 24 group, CB immunoreactivity in myenteric plexus was weaker than that of PM 12 group (Fig. 3E). In addition, the number of $\mathrm{CB}$-immunoreactive neurons decreased in PM 24 group compared to other groups except for PM 1.5 group (Fig. 2). The number of CB-immunoreactive neurons was observed into $7.7 \pm 0.5$ cells per unit area from 7 preparations (Fig. 2). However, the number of sec- ondary components in internodal strand was increased (Fig. 3E).

\section{DISCUSSION}

The ENS is similar to the central nervous system in having different types of neurons that contain a wide range of neurons specific marker substances, including many neuropeptides [8]. The immunohistochemical localization of these substances has been important for the analysis of the neuronal circuitry of the ENS [5, 6, 40]. It has been reported that the neuronal loss in the gastrointestinal tract of the rat starts in early adulthood and continues in a roughly linear manner over the rodent's lifespan [36-39].

In the present study, we examined the changes of neuron numbers per unit area and their CB immunoreactivity with aging in the myenteric plexus of gerbil duodenum. Number of myenteric neurons per unit area was similar between PM 3 to PM 12 groups. However, number of myenteric neurons of PM 1.5 group was significantly larger than that of other groups, and number of myenteric neurons of PM 24 group significantly decreased (17\%) compared to PM 3, PM 6 and PM 12 groups in the gerbil duodenum. This result is similar to the previous studies in the guinea pig $(-18 \%)$, human (34\%), mouse $(-40 \%)$ and rat (15-25\%) [12, 13, 19, 36, 40, 41].

CB-immunoreactive neurons in the duodenal myenteric plexus of gerbil duodenum were observed in all age groups, and there was no preferential location of CB-immunoreactive nerve cell bodies in the myenteric plexus. However, the number of CB-immunoreactive neurons in the myenteric plexus of gerbil duodenum showed some differences with their age. In PM 1.5, the largest number of CB-immunoreactive neurons per unit area was identified similar with overall numbers of myenteric neurons. CB-immunoreactive neuronal numbers were mostly stable throughout PM 3 to PM 12, then slightly decreased (5\%) in PM 24 compared to these groups. The proportion of CB-immunoreactive neurons per myenteric neuron in unit area was $21-24 \%$ in the gerbil duodenum. Similarly, CB-immunoreactive myenteric neurons in the small intestine of the guinea-pig were $20-30 \%$ of whole populations $[5,6,9,17]$.

We postulated that significant decrease of CB-immunoreactive neurons and overall myenteric neurons between PM 1.5 and PM 3 may be correlated with the increase of sizes of cob-web like structures and duodenal circumference during the postnatal development. In the mouse, duodenal circumferences were significantly increased concerning the thickness of muscular and submucosal propria between PM 1 and PM 3 [13]. Therefore, more ganglia per unit area could be observed in PM 1.5 group compared to that of other groups relatively. Increasing of the circumferences between PM 6 and 24 groups may be more related with the changes of whole volume of duodenum. Similar age-related increase of circumferences was reported in the mouse duodenum [13]. However, there were no statistical differences in the number of ganglia per unit area [13]. Although we identified CB- 

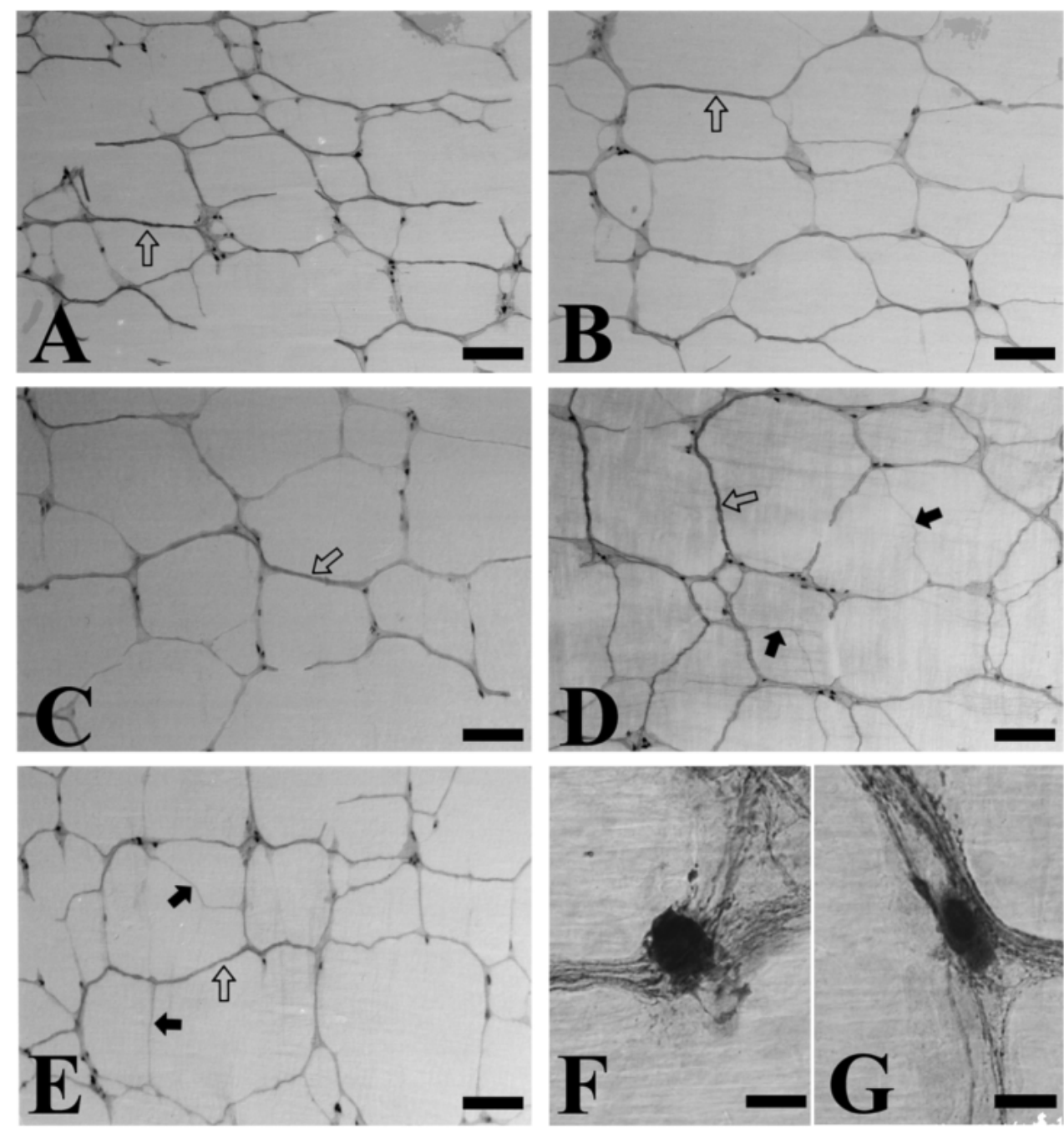

Fig. 3. Immunohistochemical staining for calbindin D-28k (CB) in the whole mount preparations of duodenum at postnatal month (PM) 1.5 (A), 3 (B), 6 (C), 12 (D) and 24 (E). In PM 1.5 group, cobweb-like structures are not fully developed in whole mount preparation (A). Cobweb-like structures are well developed from PM 3 to PM 24 groups. Some thick CB-immunoreactive internodal strands are observed in all age groups (open arrows), and fiber bundles of the secondary components of the internodal strand are increased in PM12 and 24 (arrows, D, E). High magnification views of CB-immunoreactive neurons showing Dogiel type II morphology (F and G). Bar=800 $\mu \mathrm{m}$ (A-E), $20 \mu \mathrm{m}(\mathrm{F}, \mathrm{G})$.

expressed cell loss in aged gerbil, the proportion of them is comparable to that of overall neuronal loss in the gerbil duodenum. It needs further study to clarify what types of neurons diminished in the non CB-immunoreactive neurons. Those neurons may be cholinergic or nitrergic neurons. Because, cholinergic neurons were not all immunoreactive for $\mathrm{CB}$, and nitrergic neurons also decreased in the gastrointestinal tract of aged rat and guinea-pig [43, 45].

In conclusion, we identified that $\mathrm{CB}$-immunoreactive neurons as well as overall populations of neurons were decreased in the myenteric plexus of aged gerbil duodenum. It has been described that more than $70 \%$ of $\mathrm{CB}$ expressed neurons were Dogiel type II neurons in the myenteric plexus of guinea-pig, and they were coexpressed choline acetyltransferase [5, 6, 10, 16, 26, 30, 42]. Age-related decreasing responses of cholinergic receptor, cholinergic neurotransmission and relaxation in the small intestine were reported previously $[1,2,28,32,35]$. In addition, intracellular calcium stores of smooth muscle cells of rat large intestine were affected by aging $[10,27,31,33]$. Therefore, agerelated loss of myenteric neuron may be associated with decline of the sensory or motor functions and neurodegeneration with aging in the gerbil duodenum.

ACKNOWLEGEMENT. This work supported by Research Institute of Veterinary Science. 


\section{REFERENCES}

1. Baker, D.M., Watson, S.P. and Santer, R.M. 1991. Evidence for a decrease in sympathetic control of intestinal function in the aged rat. Neurobiol. Aging 12: 363-365.

2. Bitar, K. 2003. Aging and neural control of the GI tract: V. Aging and gastrointestinal smooth muscle: from signal transduction to contractile proteins. Am. J. Physiol. Gastrointest. Liver Physiol. 284: G1-G7.

3. Bu, J., Sathyendra, V., Nagykery, N. and Geula, C. 2003. Agerelated changes in calbindin-D28k, calretinin, and parvalbumin-immunoreactive neurons in the human cerebral cortex. Exp. Neurol. 182: 220-231.

4. Buchan, A.M.J. and Baimbridge, K.G. 1988. Distribution and co-localization of calbindin D28k with VIP and neuropeptide $\mathrm{Y}$ but not somatostatin, galanin and substance $\mathrm{P}$ in the enteric nervous system of the rat. Peptides 9: 333-338.

5. Clerc, N., Furness, J.B., Bornstein, J.C. and Kunze, W.A.A. 1998. Correlation of electrophysiological and morphological characteristics of myenteric neurons of the duodenum in the guinea-pig. Neuroscience 82: 899-914.

6. Clerc, N., Furness, J.B., Li, Z.S., Bornstein, J.C. and Kunze, W.A.A. 1998. Morphological and immunohistochemical identification of neurons and their targets in the guinea-pig duodenum. Neuroscience 86: 679-694.

7. Costa, M. and Brookes, S.J.H. 1994. The enteric nervous system. Am. J. Gastroenterol. 89: 129-137.

8. Costa, M., Furness, J.B. and Gibbins, I.L. 1986. Chemical coding of enteric neurons. Prog. Brain Res. 68: 217-239.

9. Costa, M., Brookes, S.J., Steele, P.A., Gibbins, I., Burcher, E. and Kandiah, C.J. 1996. Neurochemical classification of myenteric neurons in the guinea-pig ileum. Neuroscience 75: 949967.

10. Cowen, T., Johnson, R.J.R., Soubeyre, V. and Santer, R.M. 2000. Restricted diet rescues rat enteric motor neurons from age related cell death. Gut 47: 653-660.

11. De Jong, D.I., Naber, P.A., van der Zee, E.A., Thompson, L.T., Disterhoft, J.F. and Luiten, P.G.M. 1996. Age-related loss of calcium binding proteins in rabbit hippocampus. Neurobiol. Aging 17: 459-465.

12. de Souza, R.R., Moratelli, H.B., Borges, N. and Liberti, E.A. 1993. Age-induced nerve cell loss in the myenteric plexus of the small intestine in man. Gerontology 39: 183-188.

13. El-Salhy, M., Sandstrom, O. and Holmlund, F. 1999. Ageinduced changes in the enteric nervous system in the mouse. Mech. Ageing Dev. 107: 93-103.

14. Erde, S.M., Sherman, D. and Gershon, M.D. 1985. Morphology and serotonergic innervation of physiologically identified cells of the guinea pig's myenteric plexus. J. Neurosci. 5: 617633.

15. Furness, J.B., Bornstein, J.C., Pompolo, S., Young, H.M., Kunze, W.A.A. and Kelly, H. 1994. The circuitry of the enteric nervous system. Neurogastroenterol. Motil. 6: 241-253.

16. Furness, J.B., Keast, J.R., Pompolo, S., Bornstein, J.C., Costa, M., Emson, P.C. and Lawson, D.E.M. 1988. Immunohistochemical evidence for the presence of calcium-binding proteins in enteric neurons. Cell Tissue Res. 252: 79-87.

17. Furness, J.B., Trussell, D.C., Pompolo, S., Bornstein, J.C. and Smith, T.K. 1990. Calbindin neurons of the guinea-pig small intestine: quantitative analysis of their numbers and projections. Cell Tissue Res. 260: 261-272.

18. Furness, J.B. 2000. Types of neurons in the enteric nervous system. J. Auton. Nerv. Syst. 81: 87-96.
19. Gabella, G. 1989. Fall in the number of myenteric neurons in ageing guinea-pigs. Gastroenterology 96: 1493-1497.

20. Goyal, R.K. and Hirano, I. 1996. The enteric nervous system. New Engl. J. Med. 334: 554-563.

21. Hirst, G.D.S., Holman, M.E. and Spence, I. 1974. Two types of neurons in the myenteric plexus of duodenum in the guineapig. J. Physiol. Lond. 236: 303-326.

22. Hirst, G.D.S., Johnson, S.M. and van Helden, D.F. 1985. The calcium current in a myenteric neuron of the guinea-pig ileum. J. Physiol. 361: 297-314.

23. Huguet, F., Comoy, E., Piriou, A. and Bohuon, C. 1993. Agerelated changes of noradrenergic NPY interaction in rat brain: norepinephrine, NPY levels and $\alpha$-adrenoceptors. Brain Res. 625: 256-260.

24. Hwang, I.K., Kang, T.C., Lee, I.S., Park, S.K., An, S.J., Jeong, Y.G., Seo, J.G., Oh, Y.S. and Won, M.H. 2002. Age-related change of calbindin D-28k immunoreactive neurons in the rat main olfactory bulb. Neurosci. Lett. 326: 159-162.

25. Hwang, I.K., Yoo, K.Y., Nam, Y.S., Choi, J.H., Seo, K., Lee, I.S., Jung, J.Y., Kang, T.C., Oh, Y.S. and Won, M.H. 2006. Age-related changes in calretinin-immunoreactive periglomerular cells in the rat main olfactory bulb. J. Vet. Med. Sci. 68 : 465-469.

26. Iyer, V., Bornstein, J.C., Costa, M., Furness, J.B., Takahashi, Y. and Iwanaga, T. 1988. Electrophysiology of guinea-pig myenteric neurons correlated with immunoreactivity for calcium binding proteins. J. Auton. Nerv. Syst. 22: 141-150.

27. Kawai, K., Nowak, T.S. and Klatzo, I. 1995. Loss of parvalbumin immunoreactivity defines selectively vulnerable thalamic reticular nucleus neurons following cardiac arrest in rats. Acta Neuropathol. Berl. 86: 262-269.

28. Kobashi, Y.L., Breuing, E.P. and Markus, R.P. 1985. Agerelated changes in the reactivity of the rat jejunum to cholinoceptor agonists. Eur. J. Pharmacol. 115: 133-138.

29. Lees, G.M., Makenzie, G.M. and Pearson, G.T. 1992. Complex correlations between the morphology, electrophysiology and peptide immunohistochemistry of guinea-pig enteric neurons. Eur. J. Morphol. 30: 123-136.

30. Li, Z.S. and Furness, J.B. 1998. Immunohistochemical localization of cholinergic markers in putative intrinsic primary afferent neurons of the guinea-pig small intestine. Cell Tissue Res. 294: 35-43.

31. Lopes, G.S., Mora, O.A.., Cerri, P., Jurkiewicz, N.H., Jurkiewicz, A. and Smaili, S.S. 2004. Mitochondrial alterations and apoptosis in smooth muscle from aged rats. Biochim. Biophys. Acta. 1658: 187-194.

32. Lopes, G. S., Smaili, S. S., Neto, A. C., Vladimirova, I., Jurkiewicz, A. and Jurkiewicz, N. H. 2007. Aging-induced decrease of cholinergic response and calcium sensitivity on rat jejunum contractions. J. Gerontol. A Biol. Sci. Med. Sci. 62: 264-270.

33. Mouatt-Prigent, A., Agid, Y. and Hirsch, E.C. 1994. Does the calcium binding protein calretinin protect dopaminergic neurons against degeneration in Parkinson's disease? Brain Res. 668: $62-70$.

34. North, R.A. 1973. The calcium-dependent slow after-hyperpolarization in myenteric plexus neurons with tetrodotoxin-resistant action potentials. Br. J. Pharmacol. 49: 709-711.

35. Ochillo, R.F. and Cheng, T.A. 1988. Comparative study of the effects of aging on the responsiveness of the cholinergic receptor of the isolated ileum of mouse and rat. Res. Commun. Chem. Pathol. Pharmacol. 60: 261-264.

36. Phillips, R.J., Kieffer, E.J. and Powley, T.L. 2003. Aging of the 
myenteric plexus: neuronal loss is specific to cholinergic neurons. Auton. Neurosci. 106: 69-83.

37. Phillips, R.J., Hargrave, S.L., Rhodes, B.S., Zopf, D.A. and Powley, T.L. 2004. Quantification of neurons in the myenteric plexus: an evaluation of putative pan-neuronal markers. J. Neurosci. Methods 133: 99-107.

38. Phillips, R.J., Kieffer, E.J. and Powley, T.L. 2004. Loss of glia and neurons in the myenteric plexus of the aged Fischer 344 rat. Anat. Embryol. (Berl.) 209: 19-30.

39. Phillips, R.J. and Powley, T.L. 2001. As the gut ages: timetables for aging of innervation vary by organ in the Fischer 344 rat. J. Comp. Neurol. 434: 358-377.

40. Saffrey, M.J. 2004. Ageing of the enteric nervous system. Mech. Ageing Dev. 125: 899-906.

41. Santer, R.M. 1994. Survival of the population of NADPH-diaphorase stained myenteric neurons in the small intestine of aged rats. J. Auton. Nerv. Syst. 49: 115-121.

42. Steele, P.A., Brookes, S.J. and Costa, M. 1991. Immunohistochemical identification of cholinergic neurons in the myenteric plexus of guinea-pig small intestine. Neuroscience 45: 227-239.

43. Takahashi, T., Qoubaitary, A., Owyang, C. and Wiley, J.W. 2000. Decreased expression of nitric oxide synthase in the colonic myenteric plexus of aged rats. Brain Res. 883: 15-21.

44. Timmermans, J.P., Adriaensen, D., Cornelissen, W. and Scheuermann, D.W. 1997. Structural organization and neuropeptide distribution in the mammalian enteric nervous system, with special attention to those components involved in mucosal reflexes. Comp. Biochem. Physiol. [A] 118: 331-340.

45. Wade, P.R. 2002. Aging and neural control of the GI tract. I. age-related changes in the enteric nervous system. Am. J. Physiol. Gastrointest. Liver Physiol. 283: G489-G495. 